\title{
Detection and analysis of atmospheric muons using the ALICE detector at the LHC
}

\author{
Bruno Alessandro ${ }^{\mathrm{a}}$ for the ALICE Collaboration \\ I.N.F.N. Sezione di Torino, Italy
}

\begin{abstract}
ALICE is a general purpose experiment designed to investigate nucleus-nucleus collisions at the CERN Large Hadron Collider (LHC). Located 52 meters underground, with 28 meters of overburden rock, it has also been used to detect the muonic component of the extensive air showers produced by cosmicray interactions in the upper atmosphere. A program of cosmic-ray data taking, with specific triggers for atmospheric muons, was started in 2010 in periods when there is no beam circulating in the LHC. Several million events have been recorded to date. The large size and excellent tracking capability of the ALICE Time Projection Chamber are exploited to detect and reconstruct these muons. In this paper the analysis of the multiplicity distribution of the atmospheric muons detected by ALICE between 2010 and 2013 is presented, along with the comparison with Monte Carlo simulations. Special emphasis is given to the study of high multiplicity events containing more than 100 reconstructed muons. The comprehension of the frequency of these events was an unsolved problem since the pioneering studies performed by ALEPH and DELPHI experiments at LEP. In our work the ALICE measurements show that such high multiplicity events demand primary cosmic rays with energy above $10^{16} \mathrm{eV}$. Their frequency can be successfully described by assuming a heavy mass composition of primary cosmic rays above this energy and using the most recent interaction models to describe the development of the air shower resulting from the primary interaction.
\end{abstract}

\section{Introduction}

ALICE (A Large Ion Collider Experiment) [1] is a generalpurpose, Heavy-Ion detector at the CERN LHC. It focuses on the study of the properties of the Quark-Gluon Plasma (QGP) created in strongly interacting matter at extreme energy densities in high energy nucleus-nucleus collisions. The underground location of the experiment allowed ALICE to also develop a cosmic-ray physics program, exploiting the excellent tracking capabilities of the Time Projection Chamber (TPC) to detect and reconstruct the muons produced in extensive air showers.

The use of collider detectors to study the atmospheric muons was pioneered by the LEP experiments ALEPH, DELPHI and L3. All results reported by them were consistent with standard hadronic interaction models, except the observation of high muon multiplicity events $[2,3]$. These experiments concluded that the high multiplicity events occur with a frequency which is almost an order of magnitude above expectation, even when assuming that the primary cosmic rays are purely composed of iron nuclei.

A development of this program is possible at the LHC, where the experiments are expected to operate for many years with the possibility of collecting a very large sample of cosmic-ray data. In this context ALICE began a cosmicray physics program taking data between 2010 and 2013.

Around 22.6 million events with at least 1 cosmic muon were recorded in 30.8 days of live time.

a e-mail: alessandro@to.infn.it
The analysis of these data and in particular the study of the muon multiplicity distribution, with the purpose of addressing the problem of the rate of high muon multiplicity events, is discussed in this paper.

\section{The ALICE detectors for cosmic-ray physics}

The ALICE experiment is located at Point 2 of the LHC tunnel, $52 \mathrm{~m}$ underground and with $28 \mathrm{~m}$ of overburden rock. This depth of rock completely absorbs the electromagnetic and hadronic components of the cosmic-ray induced air shower, and poses an energy threshold of $16 \mathrm{GeV}$ for vertical muons [4].

ALICE is a typical collider experiment with a cylindrical symmetry around the beam pipe. A solenoid magnet houses the central barrel detectors, where different techniques are exploited to detect and reconstruct all particles coming out from the primary interaction vertex. A forward muon arm, consisting of an absorber, a large dipole magnet and planes of gaseous detectors, is located on one side outside the solenoid magnet. A detailed description of the ALICE detector is given in [1]. The detectors used for cosmic-ray data taking are described below.

The Alice COsmic Ray DEtector (ACORDE) is an array of 60 scintillator modules located on the three top octants of the ALICE magnet, covering $10 \%$ of their surface. Each module consists of two superimposed plastic scintillators. The trigger is given by the coincidence of the signal from two different modules in a $100 \mathrm{~ns}$ window. The rate is about $1 \mathrm{~Hz}$. 
The Silicon Pixel Detector (SPD) forms the two innermost coaxial cylinders of the Inner Tracking System (ITS) around the beam pipe, centered at the nominal position of the interaction point with a radius of $39 \mathrm{~mm}$ and $76 \mathrm{~mm}$, respectively. The trigger configuration used for cosmic detection requires a coincidence between signals coming from the top and bottom halves of the outermost layer, which generated a trigger rate of around $0.16 \mathrm{~Hz}$.

The Time Of Flight (TOF) detector is a cylindrical array of 1638 Multi-gap Resistive Plate Chambers pads arranged in 18 sectors, completely surrounding the TPC. The trigger configuration used for the present analysis requires a coincidence between a signal in a sector of the upper part and a signal in either a sector of the opposite lower part forming a back-to-back alignment with respect to the central axis, or in one of the two sectors adjacent to the opposite lower sector. The rate of this trigger configuration is about $80 \mathrm{~Hz}$.

Cosmic ray data were acquired with a combination (logical OR) of at least two out of the three trigger conditions (ACORDE, SPD and TOF) depending on the run period.

The ALICE Time Projection Chamber (TPC) is the largest detector of its type, and is the main ALICE tracking device with excellent capabilities for high-track densities. The TPC has an inner radius of $80 \mathrm{~cm}$, an outer radius of $280 \mathrm{~cm}$ and a total length of $500 \mathrm{~cm}$ along the beam axis. It is filled with a mixture of $\mathrm{Ne}-\mathrm{CO}_{2}-\mathrm{N}_{2}$ and is read out by multi-wire proportional chambers at both end caps. The total area for cosmic muon detection is about $26 \mathrm{~m}^{2}$, however after applying a minimum length cut to the reconstructed muon tracks the effective area reduces to about $17 \mathrm{~m}^{2}$.

\section{Data selection and reconstruction}

The data used for the cosmic-ray analysis presented here [5] were collected between 2010 and 2013 during periods without circulating beams in LHC. Most of the data were taken with magnetic field of $0.5 \mathrm{~T}$ with both polarity, while a smaller sample was collected without magnetic field. The integrated live time amounts to 30.8 days, during which $\sim 22.6 \mathrm{M}$ events with at least 1 reconstructed muon in the TPC were accumulated. The majority of them are single muon events. A total of 7487 multi-muon events were collected, having at least 5 muons detected in the TPC.

The TPC tracking algorithm was designed to reconstruct tracks coming out from the interaction region, working inwards from the outer radius. As a consequence a cosmic muon crossing the TPC gets reconstructed as two separate tracks, called up and down, belonging to the two halves of the TPC cylinder. A specific algorithm was developed to match the two track segments as a single one. Real and Monte Carlo events of different multiplicities were used to optimize the parameters of this matching algorithm. Moreover, to avoid possible reconstruction inaccuracies associated with the most inclined tracks, the zenith angle of all events was restricted to $0<\theta<50^{\circ}$.

TPC tracks were required to consist of at least 50 clusters (out of a maximum of 159) and, in events where the magnetic field was on, to have a minimum momentum of $0.5 \mathrm{GeV} / c$, to eliminate all possible background from

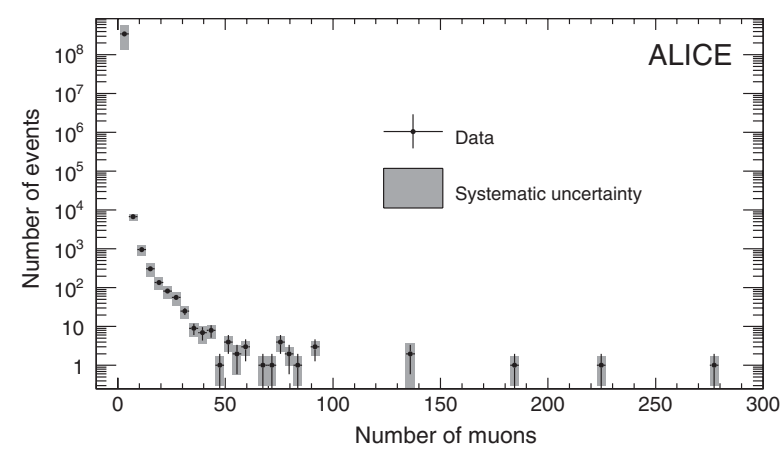

Figure 1. Atmospheric muon multiplicity distribution of the whole sample of data (2010-2013) corresponding to 30.8 days of data taking.

$e^{ \pm}$. For multi-muon events a parallelism cut was also applied, which requires the angular difference between two tracks to be $\cos \Delta \psi>0.99$ to accept the tracks. Finally, to match up and down tracks a maximum distance of closest approach of $3 \mathrm{~cm}$ in the TPC middle plane was imposed. A muon reconstructed with two TPC tracks (up and down) is called a matched muon. A track satisfying all cuts but without the counterpart in the other sector (up or down) is still accepted as a muon but flagged as a single-track muon. Most single-track muons are particles crossing the TPC near the edges where part of their trajectory may fall outside the sensitive volume.

\section{The muon multiplicity distribution}

The first measurement was the muon multiplicity distribution (MMD) to analyse its behavior and to confirm or not the existence of high muon multiplicity (HMM) events as observed by the Aleph and Delphi experiments. The MMD obtained from the whole data sample and corrected for trigger efficiency is shown in Fig. 1 [5]. The systematic uncertainties were estimated by varying the parameters of the track reconstruction and matching algorithms.

The data show a smooth distribution up to a muon multiplicity of $\sim 70$ and then 5 events with a multiplicity greater than 100. Events with $N_{\mu}>100$ are defined HMM events.

In order to understand the MMD, simulated events equivalent to 30.8 days of live time were generated using CORSIKA [6] as event generator and QGSJET [7] for the hadronic interaction model. CORSIKA 6990 with QGSJET II-03 was used to study the MMD and HMM events, CORSIKA 7350 with QGSJET II-04 was used to further check and confirm the frequency of HMM events. Two samples, pure proton (p), representing a light composition and pure iron (Fe), representing an extremely heavy compositions, were generated. The primary cosmicray energy was restricted to the interval $10^{14}<E<$ $10^{18} \mathrm{eV}$ following the usual power law energy spectrum $E^{-\gamma}$, with a spectral index $\gamma=2.7$ below the knee $\left(E_{k}=3 \times 10^{15} \mathrm{eV}\right)$ and $\gamma=3.0$ above. The total allparticle absolute flux was extracted from [8]. For each shower the core was randomly scattered at the surface level over an area $205 \times 205 \mathrm{~m}^{2}$ centered above the nominal LHC interaction point in ALICE.

The comparison between the MMD in the intermediate range $7<N_{\mu}<70$ and the simulated distribution fitted 


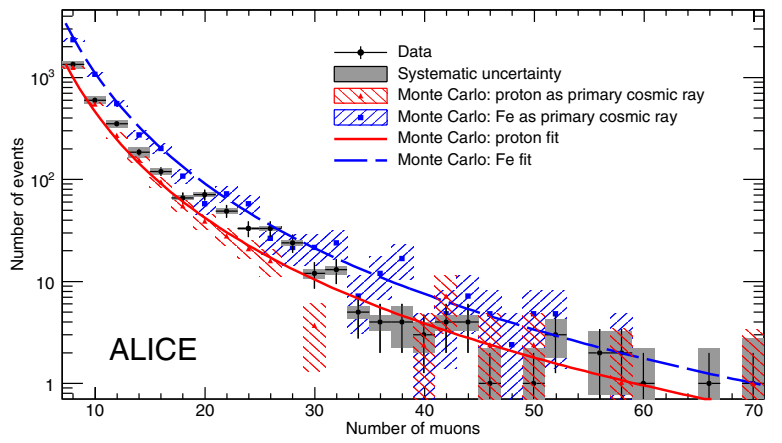

Figure 2. Measured MMD compared with values and fits obtained from simulations with $\mathrm{p}$ and $\mathrm{Fe}$ primaries.

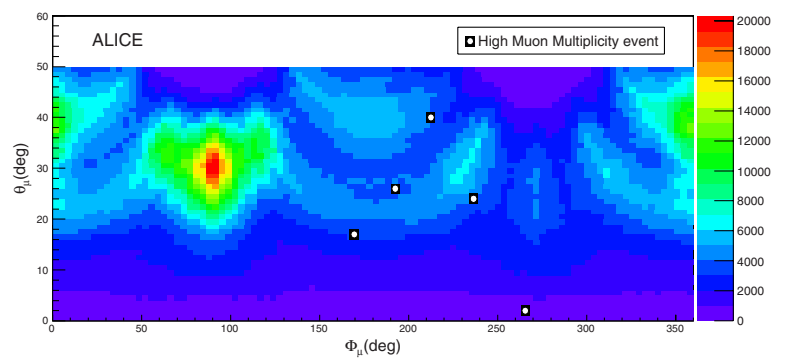

Figure 3. Zenith vs. Azimuth angle distribution of the muons in ALICE with superimposed the location of the five high muon multiplicity events (white circle on a black square).

with a power-law function is shown in Fig. 2 [5], where errors are shown separately (statistical and systematic) for data, and summed in quadrature for Monte Carlo. Below $N_{\mu} \sim 30$ the data, as expected, are between the pure $\mathrm{p}$ composition (approaching it at low multiplicity) and the pure Fe composition (at higher multiplicity). Above $\sim 30$ the low statistics does not allow to draw any firm conclusion, though the experimental points, considering their errors, are inside the region limited by the $\mathrm{p}$ and Fe curves.

\section{The high muon multiplicity events}

In 30.8 live days 5 HMM events were recorded, corresponding to a rate of $1.9 \times 10^{-6} \mathrm{~Hz}$.

To obtain a sort of radiography of the ALICE environment we have studied the angular distribution of the muons reconstructed in the TPC. Figure 3 depicts the $\theta$ (zenith angle) vs. $\Phi$ (azimuth angle) scatter plot of the arrival direction of the muons detected with the data and the location of the $5 \mathrm{HMM}$ events (white circle on a black square). It can be noted that these events do not belong to any of the areas of the access shafts (red and green zones), where the energy threshold of the muons is lower and more muons can reach the TPC.

The highest multiplicity event reconstructed in the TPC was found to contain 276 muons, corresponding to a muon areal density of $18.1 \mathrm{~m}^{-2}$. The display of this event is shown in Fig. 4.

To estimate the rate of these events, while limiting the fluctuations in the number of HMM simulated events, a live time equivalent of 1 year was simulated. A simplified Monte Carlo (which does not simulate the rock overburden and the detector response, but simply extrapolates all muon

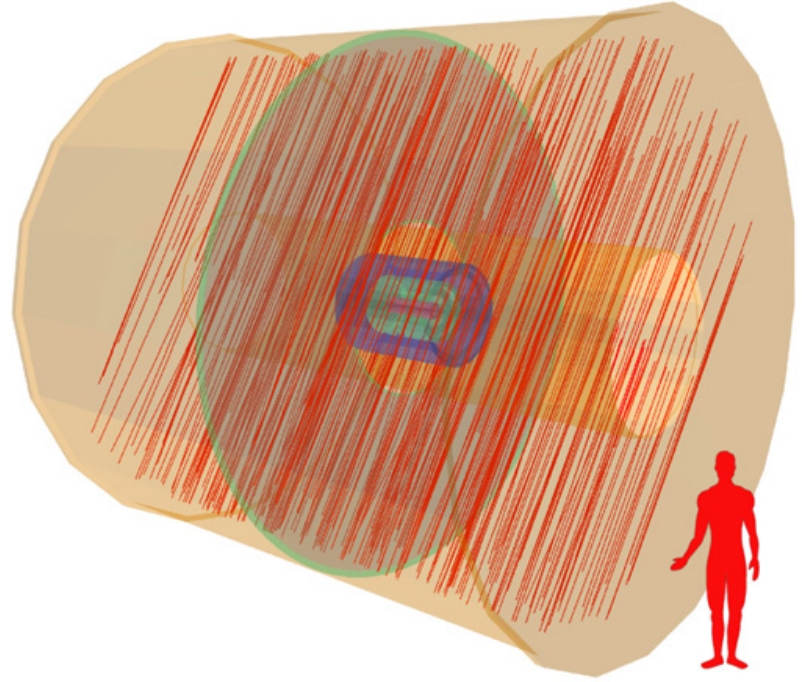

Figure 4. Event display of the highest multiplicity event found with 276 reconstructed muons crossing the TPC.

Table 1. Comparison of the HMM event rate between data and Monte Carlo.

\begin{tabular}{|l|c|c|c|c|c|}
\hline \multirow{2}{*}{ HMM events } & \multicolumn{2}{|c|}{$\begin{array}{c}\text { CORSIKA 6990 } \\
\text { QGSJET II-03 }\end{array}$} & \multicolumn{2}{c|}{$\begin{array}{c}\text { QORSIKA 7350 } \\
\text { QGSET II-04 }\end{array}$} & \multirow{2}{*}{ Data } \\
\cline { 2 - 5 } & $\mathrm{p}$ & $\mathrm{Fe}$ & $\mathrm{p}$ & $\mathrm{Fe}$ & \\
\hline \hline Period & 15.5 & 8.6 & 11.6 & 6.0 & 6.2 \\
[days per event] & & & & & \\
Rate $\left[\times 10^{-6} \mathrm{~Hz}\right]$ & 0.8 & 1.3 & 1.0 & 1.9 & 1.9 \\
$\begin{array}{l}\text { Uncertainty } \\
\text { (sys+stat) (\%) }\end{array}$ & 25 & 25 & 22 & 28 & 49 \\
\hline
\end{tabular}

tracks down to the ALICE level) demonstrated that only primaries with $E>10^{16} \mathrm{eV}$ contribute to these events. Therefore the full simulation was restricted to primaries with $10^{16}<E<10^{18} \mathrm{eV}$, still with two extreme primary compositions, pure $\mathrm{p}$ and pure Fe. To further reduce the statistical fluctuations, 5 simulations of 1 year live time each were performed, using the same EAS sample and randomly varying the shower core in the $205 \times 205 \mathrm{~m}^{2}$ area. Given that the TPC acceptance is some 3000 times smaller, this ensures that the samples are statistically independent. By averaging the 5 samples the number of HMM events in 1 year and its statistical error are estimated. The uncertainties are dominated by statistical errors on real data, and by systematic errors on Monte Carlo. There are two sources of systematic errors in the simulation, the uncertainties in the generation parameters and in the muon reconstruction algorithm. Both were carefully estimated and found to amount to $\sim 20 \%$.

In Table 1 [5] the results from Monte Carlo simulations are compared with data. The rate of HMM events can be well reproduced by the latest interaction models and a primary flux extrapolated from the direct measurements at $1 \mathrm{TeV}$. Pure Fe primary composition seems in closer agreement with measured rate, though the large uncertainty of the latter prevents a definite conclusion about the elements originating these events. This is consistent with the fact that HMM events stem from primaries with energy $>10^{16} \mathrm{eV}$, where the composition is expected to be dominated by heavier elements. 


\section{Conclusions}

In 2010-2013 the ALICE experiment collected 30.8 live days of cosmic-ray data. The MMD distribution at low and intermediate multiplicity is well reproduced by Monte Carlo simulations using CORSIKA 6990 with QGSJET II03 model. The measurements by ALICE presented here suggest a mixed ion primary cosmic-ray composition with an average mass increasing with energy. In the same period $5 \mathrm{HMM}$ events $\left(N_{\mu}>100\right)$ were recorded. The observed rate is consistent with the predictions of CORSIKA 7350 with QGSJET II-04 models using a pure Fe primary composition and energies $\mathrm{E}>10^{16} \mathrm{eV}$. For the first time the rate of HMM events has been well reproduced using conventional hadronic interaction models and reasonable primary flux model.

\section{References}

[1] K. Aamodt et al. (ALICE Coll.), JINST 3, S08002 (2008)

[2] V. Avati et al. (ALEPH Coll.), Astrop. Phys. 19, 513 (2003)

[3] J. Abdallah et al. (DELPHI Coll.), Astrop. Phys. 28, 273 (2007)

[4] B. Alessandro et al. (ALICE Coll.), J. Phys. G: Nucl. Part. Phys. 32, 1295 (2006)

[5] J. Adam et al. (ALICE Coll.), JCAP 01, 032 (2016)

[6] D. Heck et al. FZKA-6019 (1998)

[7] S. Ostapchenko, Nucl. Phys. Proc. Suppl. 151, 143 (2006)

[8] J. R. Hörandel, Astrop. Phys. 19, 193 (2003) 